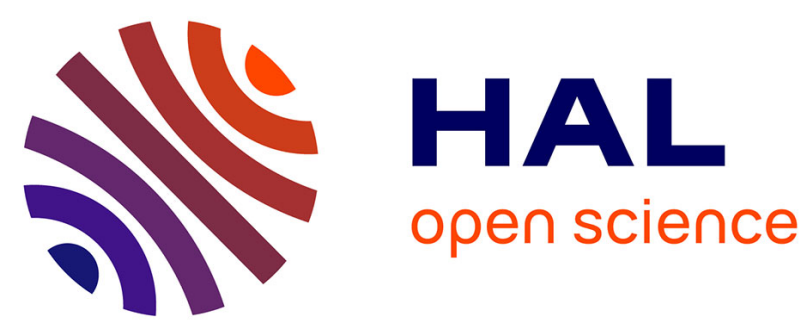

\title{
Investigation of radiation temperature and straining temperature effects on the screw dislocation mobility evolution in irradiated ferritic grains using 3D dislocation dynamics
}

\author{
Y. Li, C. Robertson, X.F. Ma, Biao Wang
}

\section{To cite this version:}

Y. Li, C. Robertson, X.F. Ma, Biao Wang. Investigation of radiation temperature and straining temperature effects on the screw dislocation mobility evolution in irradiated ferritic grains using 3D dislocation dynamics. TMS Annual Meeting and Exhibition - 2019, Mar 2019, San Antonio, United States. cea-02338715

\section{HAL Id: cea-02338715 https://hal-cea.archives-ouvertes.fr/cea-02338715}

Submitted on 24 Feb 2020

HAL is a multi-disciplinary open access archive for the deposit and dissemination of scientific research documents, whether they are published or not. The documents may come from teaching and research institutions in France or abroad, or from public or private research centers.
L'archive ouverte pluridisciplinaire HAL, est destinée au dépôt et à la diffusion de documents scientifiques de niveau recherche, publiés ou non, émanant des établissements d'enseignement et de recherche français ou étrangers, des laboratoires publics ou privés. 


\title{
Investigation of radiation temperature and straining temperature effects on the screw dislocation mobility evolution in irradiated ferritic grains using 3D dislocation dynamics
}

\author{
Y. Li ${ }^{\text {a,b, }}$, C. Robertson ${ }^{\text {a }}$, X. F. Ma ${ }^{\text {b }}$, B. Wang ${ }^{\text {b }}$ \\ ${ }^{a}$ DEN-Service de Recherches Métallurgiques Appliquées, CEA, Université Paris-Saclay, F91191, \\ Gif-sur-Yvette, France \\ ${ }^{b}$ Sino-French Institute of Nuclear Engineering and Technology, Sun Yat-Sen University, 519082, \\ Zhuhai, China
}

\section{Abstract}

Nuclear structural materials subjected to neutron irradiation accumulate dose-dependent, disperse defect clusters populations. Subsequent dislocation/defect interactions induce material mechanical property degradations, including hardening and embrittlement. Our goal in this work is to evaluate the effect of disperse defect clusters population on the effective dislocation mobility in ferritic Fe-Cr grains, using 3D dislocation dynamics simulations. The defect induced changes of the grain-scale mechanical response are evaluated using the recently proposed Defect-Induced Apparent Temperature (DIAT) shift concept. It is found that the DIAT shift associated with a given defect dispersion scales with the ductile to brittle transition temperature (DBTT) shift associated with exactly the same, observed defect population. The dose-dependent evolutions associated with broad irradiation temperature and straining temperature changes are investigated herein, for further exploration and validation of the DIAT shift concept.

\section{Keywords: 3D dislocation dynamics simulation; DIAT shift; DBTT shift; temperature effect;}

\footnotetext{
${ }^{*}$ Correspondence author

Email address: yang.li@cea.fr $(Y . L i)$.
} 


\section{Introduction}

Reactor Pressure Vessel (RPV) steels subjected to neutron irradiation accumulate dosedependent defect cluster populations [1][2]. This effect significantly influences the lifetime and serviceability of nuclear power plants (NPP), through its impact on dislocationmediated plastic deformation mechanisms. Namely mobile dislocations strongly interact with the radiation-induced loops, as observed in TEM [3][6] and predicted by various simulation methods [5][7]. More recently, the statistical evolutions of the screw dislocation populations in presence of the radiation induced defects has been evaluated using three dimensional dislocation dynamics simulations [9]. These investigations have shown that the influence of disperse defect populations on the effective dislocation mobility can be characterized by a scalar indicator called the Defect-Induced Apparent Temperature (DIAT) shift. Interestingly, the DIAT shift magnitude and the radiation-induced evolution of the Ductile-Brittle Transition Temperature (DBTT) shift can be associated with exactly the same defect dispersion. Our goal in this work is to investigate the DIAT shift sensitivity on the irradiation temperature and the straining temperature, in relation with the corresponding DBTT shift evolutions.

\section{Model and method descriptions}

\subsection{Dislocation mobility rules and cross-slip algorithm}

The 3D dislocation dynamics simulations are performed using TRIDIS code, whereby the dislocation lines are treated as discrete, orthogonal edge and screw segments [10]. In BCC materials (such as ferritic RPV steel), the edge dislocation segment velocity writes:

$$
v_{\text {edge }}=\frac{\tau^{*} b}{B}
$$


where $\tau^{*}$ is the effective resolved shear stress, $b$ the magnitude of Burgers vector and $B$ the viscous drag coefficient. Screw dislocation mobility however depends on the thermal activated generation of kink-pairs, as sketched in Fig. 1. The kinks subsequently glide away from each other, until the screw dislocation propagates to the next Peierls valley. Screw dislocation mobility associated with the thermally activated nucleation of kink pairs depends on the «kink-pair nucleation rate per unit length», which writes [11]:

$$
J\left(\tau^{*}, T\right)=\frac{8 \pi\left(\tau^{*}\right)^{2}}{\mu B h} \exp \left(-\frac{\Delta H_{0}}{k_{B} T}\left[1-\left(\frac{\tau^{*}}{\tau_{0}}\right)^{p}\right]^{q}\right)
$$

where $h$ is the Peierls valley width, $\mu$ is the shear modulus, $k_{B}$ the Boltzmann's constant, $T$ is the temperature, and $\Delta H_{0}$ the kink-pair formation enthalpy. Parameters $p$ and $q$ characterize the shape of the thermal activation barrier with respect to $\tau^{*}$ variations, thus the term in the exponential bracket represents the total kink-pair formation enthalpy $\Delta G$. Accounting for subsequent kink pair propagation at finite velocity allows expressing the screw dislocation velocity as:

$$
v_{\text {screw }}=h J X^{\prime}=h J \frac{X_{\infty} L}{X_{\infty}+L}
$$

where the last term in Eq. (3) represents the glide distance of the kink pair along a finitelength screw dislocation, before its annihilation with a kink pair of opposite sign. Quantity $L$ is the dislocation length and $X_{\infty}$ is the kink-pair propagation distance along an infinitelong screw dislocation.

The present simulations carried out herein are adapted to $\mathrm{Fe}-\mathrm{Cr}$ grains, through the material dependent parameters as listed in Tab. 1. This kink-pair model ensures a progressive stressvelocity transition, with increasing straining temperature. The screw cross-slip mechanism is implemented using the stochastic procedure as described in [12]. It can be shown that the present DD calculations generate stress-strain evolutions compatible with $\mathrm{Fe}-\mathrm{Cr}$ steels grains for all straining temperatures, within the ductile to brittle temperature range. 


\begin{tabular}{ccccccccc}
\hline$\Delta H_{0}$ & $\boldsymbol{\tau}_{\boldsymbol{0}}$ & $\boldsymbol{p}$ & $\boldsymbol{q}$ & $\begin{array}{c}\text { Viscous drag } \\
\text { coefficient } \\
\left(\mathbf{1 0}^{-\mathbf{2 0}} \mathbf{J}\right)\end{array}$ & $\begin{array}{c}\text { Burgers } \\
\text { vector }\end{array}$ & $\begin{array}{c}\text { Young } \\
\text { modulus }\end{array}$ & $\begin{array}{c}\text { Poisson } \\
\text { ration } \boldsymbol{v}\end{array}$ & $\begin{array}{c}\text { Time } \\
\text { step }\end{array}$ \\
\hline 4.86 & 358 & 0.5 & 1 & 34 & 2.54 & 210 & 0.3 & $\Delta t(\mathbf{1 0})$ \\
& & & & & & & & \\
\hline
\end{tabular}

Table 1. Material-dependent parameters associated with Fe-Cr grains [14].

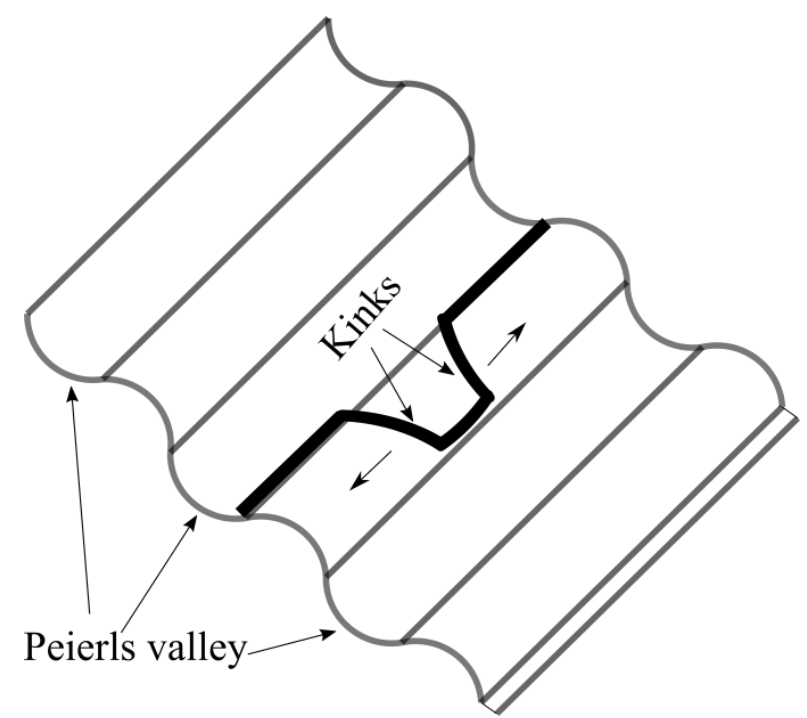

Fig. 1 Screw dislocation displacement mechanism in BCC crystals. The dislocation moves by nucleation and subsequent propagation of kink pairs.

\subsection{Simulation cases and DIAT shift concept}

The simulation cell adopted in this work is a $1 \mu \mathrm{m}^{3}$ sized cubical grain. The outer grain interfaces are impenetrable for dislocation segments, which allows for a realistic description of intra-granular stress (e.g. strain localization, for example) [13][15]. The initial dislocation sources belong to the a/2 $\left(\begin{array}{lll}1 & 0 & 1\end{array}\right)<-1 \quad-1 \quad 1>\left(\right.$ associated with a/2 $\left(\begin{array}{lll}1 & -1 & 0\end{array}\right)<-$ $\begin{array}{lll}1 & -1 & 1>\text { and } a / 2\end{array}\left(\begin{array}{lll}0 & 1 & 1\end{array}\right)<-1 \quad-1 \quad 1>$ slip systems $)$ slip system. Plastic deformation of the grain 
is carried out under the strain rate controlled conditions $\left(\dot{\varepsilon}=100 \mathrm{~s}^{-1}\right)$, with a tensile load applied along the (100) uni-axial tension direction. The radiation induced defects (interstitial dislocation loops, mainly) are implemented in the form of a population of soft planar interfaces called facets, oriented normal to the (100) direction. The facet strength is calibrated based on the MD simulations, as explained in [15]. Detailed validation of this simplified dislocation/defect implementation has been presented elsewhere [16][17]. It is known that the defect dispersions are strongly influenced by the radiation condition. Therefore varying the defect number density $N$ and the defect size $D$ allow us for investigating the radiation temperature effect on the dislocation mobility evolutions.

At fixed straining temperature, the (screw) dislocation mobility is significantly affected (reduced) by the presence of the disperse defects. Such evolution can be evaluated quantitatively using the DIAT shift concept as explained in [9]. This idea is based on observation: the (screw) dislocation mobility is influenced by both the straining temperature and the dislocation/defect interactions. In other words, the addition of dispersed defects in a pristine grain or crystal yields exactly the same (global) effect as depressing the straining temperature by a certain, definite amount. This apparent straining temperature shift is defined as DIAT shift, which turns to closely reflect the DBTT shift evolutions obtained for a broad range of disperse defect population conditions (see [9][18]).

The $\triangle$ DIAT calculation procedure is briefly explained below. Firstly, the defect-free grain is strained at temperature $T_{0}$ to obtain the average, reference screw dislocation motion $\left(\overline{\tau_{0}^{*}}, \overline{v_{0}}\right)$. Next, the irradiated grain is strained up to the same plastic strain level at the same temperature $T_{0}$. Then the deviation of $\left(\tau_{1}^{*}, v_{1}\right)$ combinations from the $\left(\overline{\tau_{0}^{*}}, \overline{v_{0}}\right)$ for each screw dislocation segment can be calculated as follows:

$$
\frac{\overline{v_{0}}}{v_{1}}\left(\frac{\tau_{1}^{*}}{\overline{\tau_{0}^{*}}}\right)^{3 / 2}=\exp \left(\frac{\Delta G_{1}\left(\tau_{1}^{*}\right)}{2 k_{B} T_{1}}-\frac{\Delta G_{0}\left(\overline{\tau_{0}^{*}}\right)}{2 k_{B} T_{0}}\right)
$$

It is important to note that the dislocation mobility rule is not changed for irradiated and unirradiated grain and therefore, the evolution of the dislocation motion is entirely caused by 
the implemented defect dispersions. A temperature shift $\left(T_{0}-T_{1}\right)$ is then obtained for each (screw) dislocation segment present in the simulated volume, yielding a single $\triangle \mathrm{DIAT}$ value. In the next section, the radiation temperature effect and the straining temperature on the DIAT shift are examined and further discussed.

\section{Results and discussions}

\subsection{Radiation temperature effect}

The fracture response (i.e. the DBTT shift amplitude) of Fe-Cr materials strongly depends on the irradiation temperature, for a fixed irradiation dose condition. Changing the irradiation temperature indeed affects the defect dispersion characteristics, in terms of defect size $D$ and defect number density $N$. For instance, the defect number density sharply decreases with increasing the irradiation temperature, whereas the loops sizes follow the opposite trend (see Tab. 2). The $\triangle \mathrm{DIAT}$ associated various defect dispersion conditions are calculated as explained in section 2.2 (at $T_{0}=300 \mathrm{~K}$ ) and presented in Fig. 2.

\begin{tabular}{ccccc}
\hline $\boldsymbol{T}_{\text {irra }}$ & $\mathbf{2 8 0}^{\circ} \mathbf{C}$ & $\mathbf{3 0 0}^{\circ} \mathbf{C}$ & $\mathbf{3 4 0}^{\circ} \mathbf{C}$ & $\mathbf{3 8 0}^{\circ} \mathbf{C}$ \\
\hline $\boldsymbol{N}\left(\mathbf{1 0}^{\mathbf{1 8}} \mathbf{m}^{\mathbf{3}}\right)$ & 6000 & 4000 & 1600 & 700 \\
$\boldsymbol{D}(\mathbf{n m})$ & 10 & 12 & 15 & 20 \\
\hline
\end{tabular}

Tab. 2 Defect number density and loop sizes of $\mathrm{Fe}-\mathrm{Cr}$ at different irradiation temperatures, for irradiation doses up to 5dpa [19][21]. 


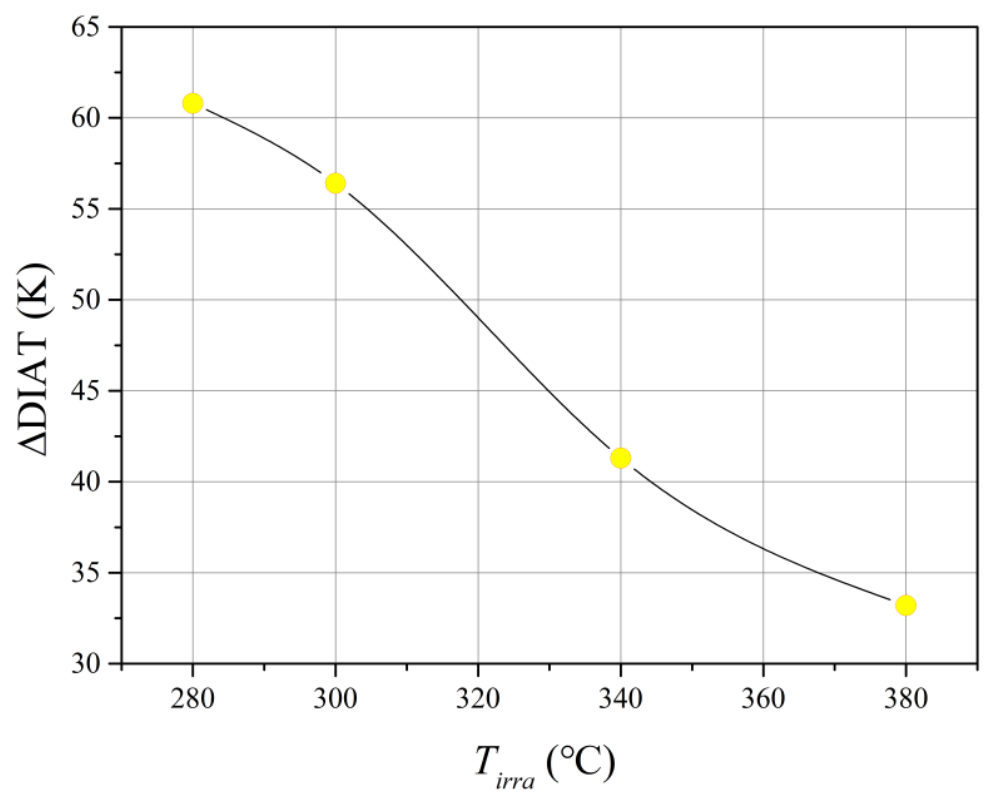

Fig. 2 DIAT shift evolution with the irradiation temperature in $\mathrm{Fe}-\mathrm{Cr}$ grains.

The DIAT shift decreases with rising the irradiation temperature, up to $T_{i r r a}=380^{\circ} \mathrm{C}$. The corresponding $\triangle \mathrm{DBTT}$ evolutions present exactly the same trend [2] (Figure 5), at least up to 1 dpa dose (Figure 6, [22]). In these conditions, it is found that the $\Delta$ DIAT magnitude and its evolutions reflect the evolutions of quantity $\sqrt{N D}$ (see Tab. 2). The $\Delta$ DIAT/ $\triangle \mathrm{DBTT}$ correlations are further explored and discussed in the next sections.

\subsection{Straining temperature effect}

Defect-free grains are simulated under three selected straining temperatures $T_{0}=250 \mathrm{~K}$, $300 \mathrm{~K}$ and $350 \mathrm{~K}$ (in DBT range). The corresponding stress-stain cures are presented in Fig. 3(a), showing a pronounced applied stress amplitude decreases with increasing temperature. The corresponding dislocation density evolutions are shown in Fig. 3(b): the total dislocation density linearly augments with the accumulation of plastic strain. The 
dislocation accumulation rate increases with straining temperature. For instance, the dislocation density at $\varepsilon_{p}=0.0012$ with $T_{0}=350 \mathrm{~K}$ is almost 2 times the value achieved at $T_{0}$ $=250 \mathrm{~K}$. This means that higher straining temperature induces faster dislocation multiplication rate, thus associated with increased dislocation activity. The present results are in good agreements with experimental observations [23], DD [24] and FEM [25] simulation results.

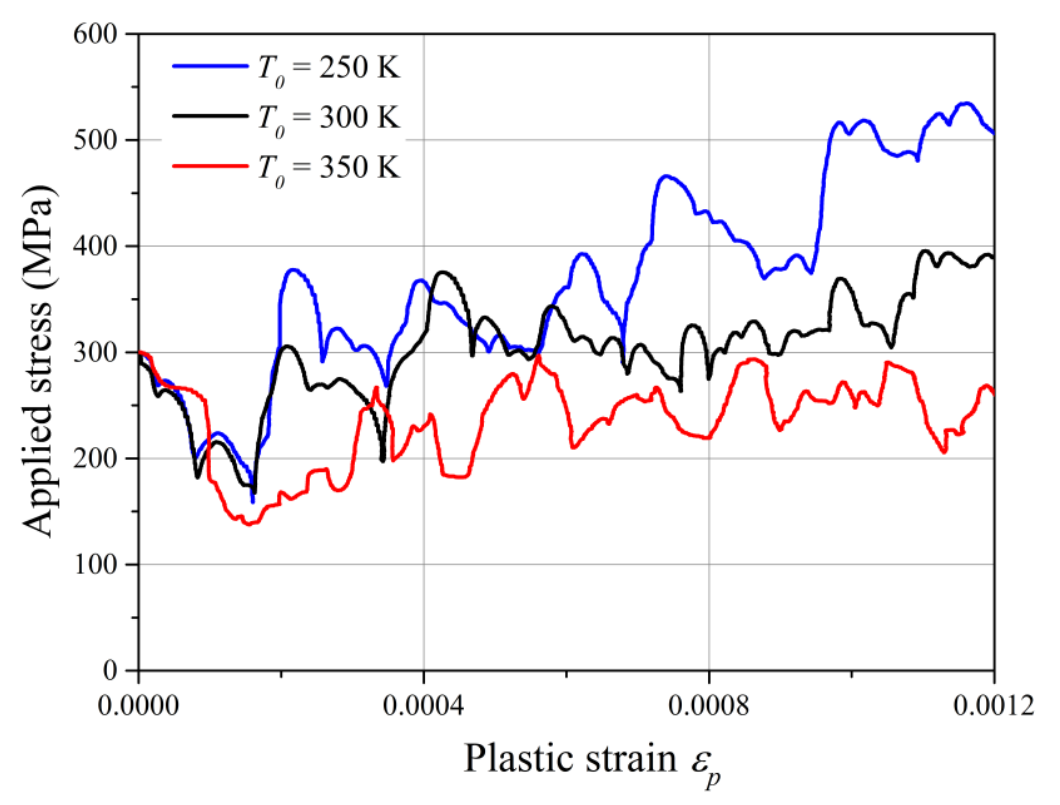

(a) 


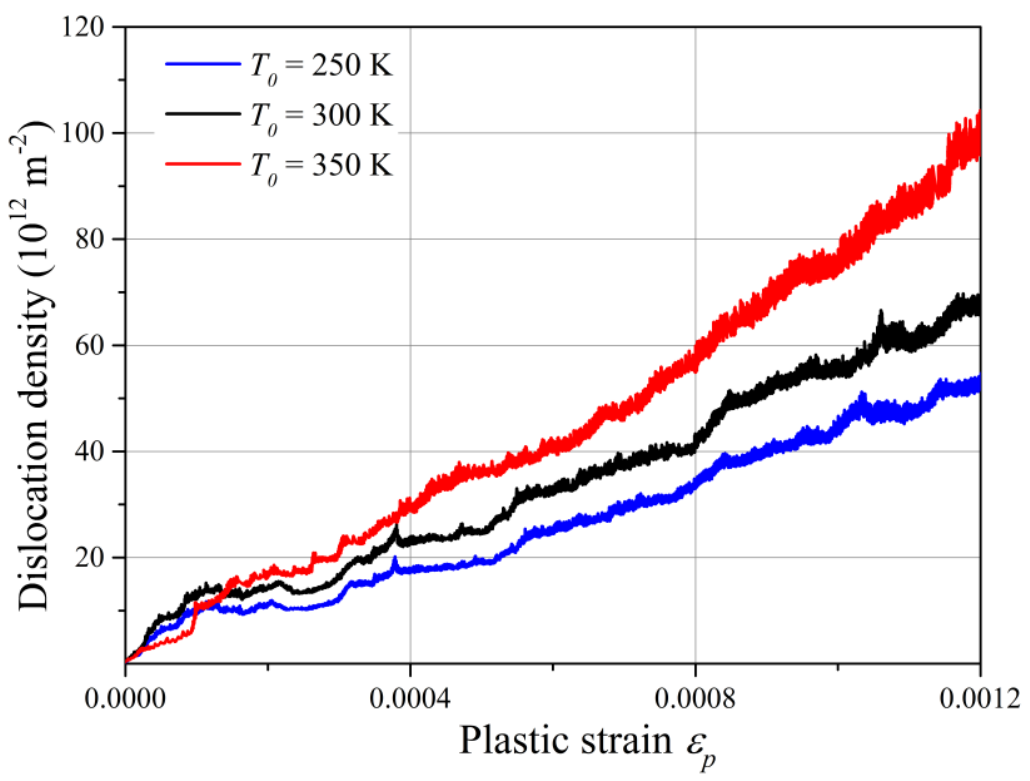

(b)

Fig. 3 Plastic strain spreading effect at different temperatures. (a) Stress-stain response. (b) Dislocation density versus plastic strain.

At each fixed straining temperature, we then introduce various defect dispersions into the grains, with a view to investigate the corresponding dislocation mobility evolutions, focusing on $D=15 \mathrm{~nm}$ loop size (corresponding to the radiation temperature around $300^{\circ} \mathrm{C}$ ) and defect number density $N=1000 \sim 5000 \times 10^{18} \mathrm{~m}^{-3}$ (corresponding to the dose in the range of $1 \sim 4 \mathrm{dpa})$ case. The $\triangle \mathrm{DIAT}$ evolutions obtained with different straining temperatures are shown in Fig. 4. At $T_{0}=250 \mathrm{~K}$, the $\triangle \mathrm{DIAT}$ magnitude almost linearly increases with respect to the defect number density. In the other 2 cases, the $\triangle \mathrm{DIAT}$ increases from $N=1000 \times 10^{18} \mathrm{~m}^{-3}$ to $N=3000 \times 10^{18} \mathrm{~m}^{-3}$ and then is saturates around $80 \mathrm{~K}$ and $90 \mathrm{~K}$, respectively. For a fixed defect number density $N$, the $\triangle \mathrm{DIAT}$ magnitude increases with the straining temperature, especially for $N>1000 \times 10^{18} \mathrm{~m}^{-3}$. 


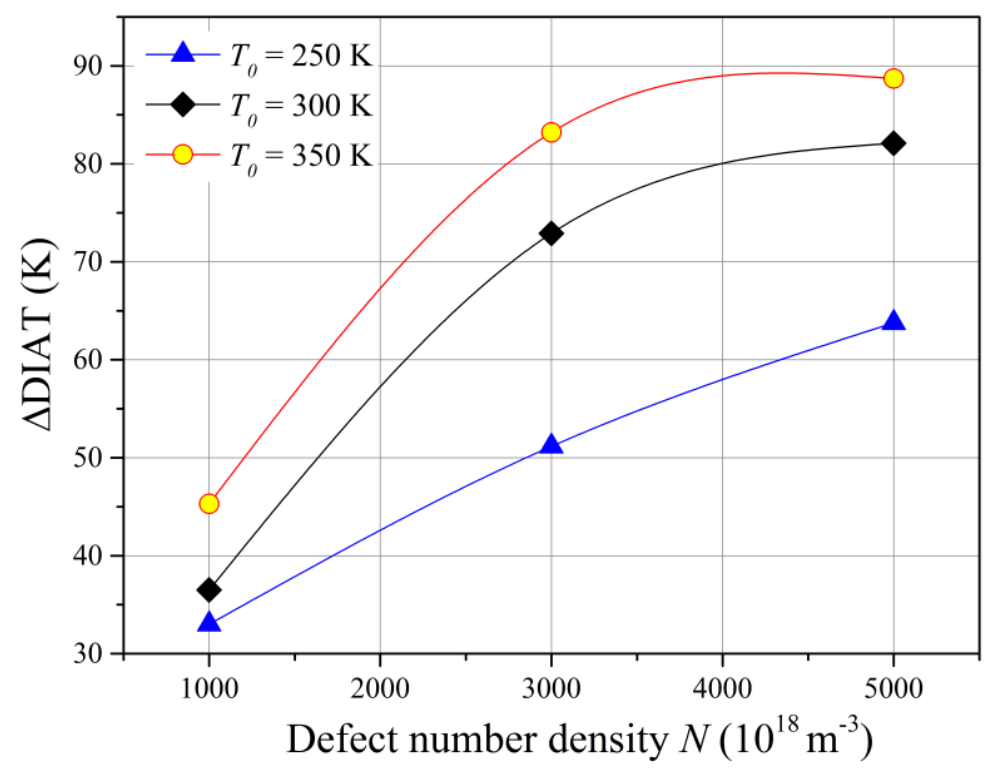

Fig. $4 \triangle$ DIAT as functions of the defect number density $N$, for different straining temperatures.

Interestingly, the $\triangle \mathrm{DIAT}$ evolutions with straining temperature closely reflect the corresponding fracture toughness response evolutions as shown in Fig. 5(a). Namely the impact energy shift increases with the testing temperature $\left(T_{0}>T_{0}{ }^{\prime}\right)$. This evolution is consistent with enhanced multiplication and propagation of dislocation populations near the fracture surface of the tested specimens. This effect directly relates to rate of dislocation/defect interaction as evaluated during the DD simulations. In the $N=3000 \times$ $10^{18} \mathrm{~m}^{-3}$ case for example, the dislocation/defect interaction count at $T_{0}=350 \mathrm{~K}$ is almost four times higher than at $T_{0}=250 \mathrm{~K}$ (for a fixed $\varepsilon_{p}=10^{-3}$ ). The (screw) dislocation population activity is then more pronounced at higher straining temperature, where the presence of defect dispersion has a larger relative impact, in terms of dislocation mobility, as shown in Fig. 5(b). This situation explains that $\left(T_{0}-T_{1}\right)>\left(T_{0}{ }^{\prime}-T_{1}{ }^{\prime}\right)$ for $T_{0}>T_{0}$ ' and hence, a DIAT shift increases with the straining temperature. 

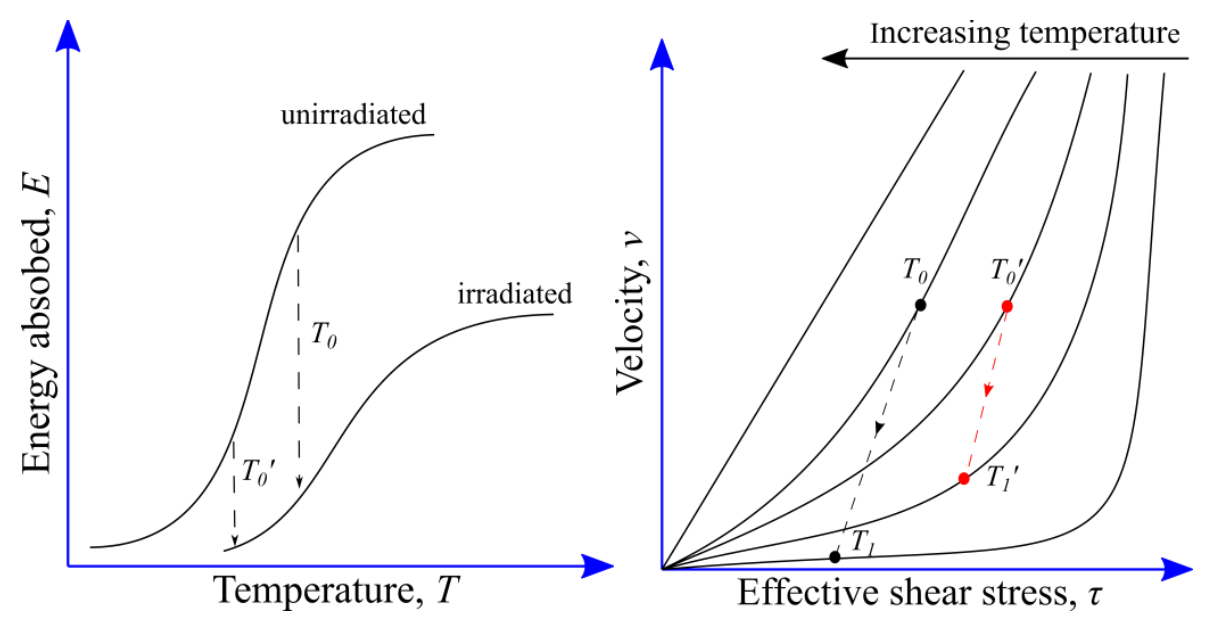

Fig. 5 Straining temperature effect. (a) Typical resilience curves obtained in un-irradiated and irradiated materials, with $T_{0}>T_{0}$ '. (b) Screw dislocation velocity versus the effective resolved shear stress, for different straining temperatures. Subscript 0 represents the dislocation motion in unirradiated grain and subscript 1 indicates the irradiated case.

We have shown that the influence of a given defect dispersion (characterized by $N$ and $D$ ) on dislocation mobility increases with the straining temperature, as does the ductile to brittle transition temperature shift associated with exactly the same $N$ and $D$ case. This comparison indicates that the DIAT shift concept can potentially predict the dosedependent DBTT evolutions associated with a broad range of irradiation conditions.

\section{Conclusion}

Plastic deformation of the $\mathrm{Fe}-\mathrm{Cr}$ cubic grains has been simulated using a 3D dislocation dynamics modelling approach, including thermally activated dislocation mobility rules. The effect of disperse defect populations on the effective (screw) dislocation mobility has been evaluated using the statistical, DIAT shift indicator. At fixed straining temperature, the 
$\triangle \mathrm{DIAT}$ magnitude decreases with the irradiation temperature, as does the $\triangle \mathrm{DBTT}$ trend associated with the exactly the same irradiation temperature evolution. For a fixed irradiation temperature (i.e. for a fixed disperse defect population $N$ and $D$ case), the $\triangle$ DIAT level increases with the straining temperature, in agreement with the corresponding resilience test results $(\triangle \mathrm{DBTT})$, due to exactly the same straining temperature evolution. The results presented in this paper show that the DIAT shift concept can potentially predict the dose-dependent DBTT shift evolutions associated with a broad range of irradiation and testing conditions.

\section{Acknowledgements}

The support of the Materials Research Program RMATE of the Nuclear Energy Division of the French Atomic Energy Commission (CEA/DEN) is also acknowledged.

Adding some Chinese fundings??? 


\section{References}

[1] Nikolaev YA, Nikolaeva AV, Shtrombakh YI (2002) Radiation embrittlement of low-alloy steels. Int. J. Pres. Ves. Pip. 79: 619-636

[2] Victorian M et al. (2000) The microstructure and associated tensile properties of irradiated fcc and bcc metals. J. Nucl. Mater. 276: 114-122.

[3] Gaganidze E, Aktaa J (2013) Assessment of neutron irradiation effects on RAFM steels. Fusion Eng. Des. 88: 118-128.

[4] Robach JS et al. (2003) In-situ transmission electron microscopy observations and molecular dynamics simulations of dislocation-defect interactions in ion-irradiated copper. Phil. Mag. 83: 955-967.

[5] Zinkle SJ, Matsukawa Y (2004) Observation and analysis of defect cluster production and interactions with dislocations. J. Nucl. Mater. 329: 88-96.

[6] Byun TS, Hashimoto N (2006) Strain localization in irradiated Materials. Nucl. Eng. Technol. 38: 619639.

[7] Kwon J, Kwon SC, Hong JH (2003) Prediction of radiation hardening in reactor pressure vessel steel based on a theoretical model. Ann. Nucl. Energy 30: 1549-1559.

[8] Bacon DJ, Osetsky YN (2005) Modelling dislocation-obstacle interactions in metals exposed to an irradiation environment, Mater. Sci. Eng. A 400: 353-361.

[9] Li Y, Robertson C (2018) Irradiation defect dispersions and effective dislocation mobility in strained ferritic grains: a statistical analysis based on 3D dislocation dynamics simulations. J. Nucl. Mat. 504: 84-93.

[10] Verdier M, Fivel M, Groma I (1998) Mesoscopic scale simulation of dislocation dynamics in fcc metals: principles and applications. Modell. Simul. Mater. Sci. Eng. 6: 755.

[11] Gilbert MR, Queyreau S, Maroa J (2011) Stress and temperature dependence of screw dislocation mobility in $\alpha$-Fe by molecular dynamics. Phys. Rev. B 84: 174103.

[12] Chaussidon J et al. (2008) Dislocation dynamics simulations of plasticity in Fe laths at low temperature. Acta Mater. 56: 5466-5476.

[13] Robertson C, Gururaj K (2011) Plastic deformation of ferritic grains in presence of ODS particles and irradiation-induced defect clusters: A 3D dislocation dynamics simulation study. J. Nucl. Mater. 415: $167-178$. 
[14] Bohmert J, Muller G (2002) Thermally activated deformation of irradiated reactor pressure vessel steel. J. Nucl. Mater. 301: 227-232.

[15] Gururaj K, Robertson C, Fivel M (2015) Post-irradiation plastic deformation in bcc Fe grains investigated by means of 3D dislocation dynamics simulations, J. Nucl. Mater. 459: 194-204.

[16] Gururaj K, Robertson C, Fivel M (2015) Channel formation and multiplication in irradiated FCC metals: a 3D dislocation dynamics investigation. Phil. Mag. 95m 1368-1389.

[17] Li Y et al. (2018) Screw dislocation interaction with irradiation defect-loops in $\alpha$-iron: evaluation of cross-slip effect using dislocation dynamics simulations. Modell. Simul. Mater. Sci. Eng. 26: 055009.

[18] Singh K et al. (2018) Irradiation effect on stress distribution in ferritic materials using dislocation based model. ICONS2018 Proceeding. In press.

[19] Matijasevic M, Lucon E, Almazouzi A (2008) Behavior of ferritic/martensitic steels after $\mathrm{n}$-irradiation at 200 and $300^{\circ}$ C. J. Nucl. Mater. 377: 101-108.

[20] Kuleshova EA et al. (2002) Comparison of microstructural features of radiation embrittlement of VVER-440 and VVER-1000 reactor pressure vessel steels. J. Nucl. Mater. 300: 127-140..

[21] Porollo SI et al. (1998) The microstructure and tensile properties of $\mathrm{Fe}-\mathrm{Cr}$ alloys after neutron irradiation at $400 \mathrm{C}$ to 5.5-7.1 dpa. J. Nucl. Mater. 256: 247-253.

[22] Kohyama et al. (1996) Low-activation ferritic and martensitic steels for fusion application. J. Nucl. Mater. 233: 138-147.

[23] Kuramoto et al. (1979) Thermally activated slip deformation of high purity iron single crystals between 4.2K and 300 K. Scripta Metall. 13: 1039-1042.

[24] Chaussidon J, Robertson C, Fivel M (2010) Internal stress evolution in Fe laths deformed at low temperature analyzed by dislocation dynamics simulations, Modell. Simul. Mater. Sci. Eng. 18: 025003.

[25] Singh K, Robertson C, Bhaduri AK (2017) Assessing the Irradiation Defect Induced Changes using Dislocation Based Crystal Plasticity Model for BCC Materials, Proc. Struct. Integrity 5: 294-301. 